\title{
Simple Pin-Plate Electrode Configuration for Targeted Electroporation
}

\author{
Ayoola Brimmo ${ }^{1}$, Mohammad Qasaimeh ${ }^{1}$ and Anoop Menachery*2 \\ ${ }^{1}$ Division of Engineering, New York University Abu Dhabi, Abu Dhabi, UAE \\ ${ }^{2}$ Institute of Engineering and Transport, Electrical and Electronics, Malta College of Arts, Science and Technology, Malta \\ *anoop.menachery@mcast.edu.mt
}

\begin{abstract}
Here, a simple pin-plate electrode setup has been constructed to reliably electroporate biological cells within droplets. The process constitutes temporary permeabilization of the plasma membrane by creating high electric fields at the tip of commercially available tapered tungsten electrodes. Subsequently, various electrical field frequencies were tested to quantify the insertion and release of dye molecules through the transient pores. Using optimized settings, we have also successfully managed to insert a plasmid to induce fluorescent protein expression, via a process referred to as transfection. The proposed design overcomes technological disadvantages of conventional cuvette-based electroporation, by creating a rapid sequentially addressable open-fluidic platform requiring small fluid volumes capable of handling multiple reagents.
\end{abstract}

Keywords: bioelectronics, electroporation, transfection, microfluidics

\section{Introduction}

The capacity to transfer DNA and RNA into cells in order to alter their genetic signature or phenotype, by a process called transfection, is essential in biological sciences [1]. Various types of transfection methods exist, of which, electroporation is an extremely common technique. Electroporation is a non-contact method that uses electrical pulses to generate high electrical fields in order to permeabilize the cell membrane barrier. It is a very effective approach for delivering DNA and other molecules to accomplish good transfection efficiencies in bacterial and mammalian cells [2].

However, common commercial electroporation devices comprise parallel plate electrodes spaced millimeters apart with a volumetric capacity of hundreds of microliters. As a result, the conventional setup utilizes relatively large amounts of transfection reagents and DNA which can prohibitively expensive. The transfection efficiency and cell viability achieved by these setups also remain low due to their limitations in performing parametric optimizations via live monitoring [3]. Some of the proposed approaches using microfluidic technology aim to counter these challenges by carrying out electroporation in enclosed microfluidic channels subject to fluid flow. However, this approach also comes along with a number of challenges, such as, a complex experimental setup containing fluidic channels, tubing and fluid pumps [4].

Our proposed open microdroplet system, shown in Fig 1, enables continuous microscopy-based monitoring of the electroporation process through the transparent bottom substrate. To establish the required electric field, the underlying substrate is made conductive by indium tin oxide coatings, which also allow cells to be cultured directly on them. The demonstrated pin-plate electrode design can accommodate an array of droplets on a single chip and is hence ideal as an experimental optimization platform for multiple electroporation reagents. Furthermore, targeted electroporation was achieved using this electrode system that provides rapid electric field decay with increasing distance from the pin electrode. Using this setup, two/three cell clusters and even single cells can be electroporated without affecting the neighbouring cells within a dense $2 \mathrm{D}$ cluster. 


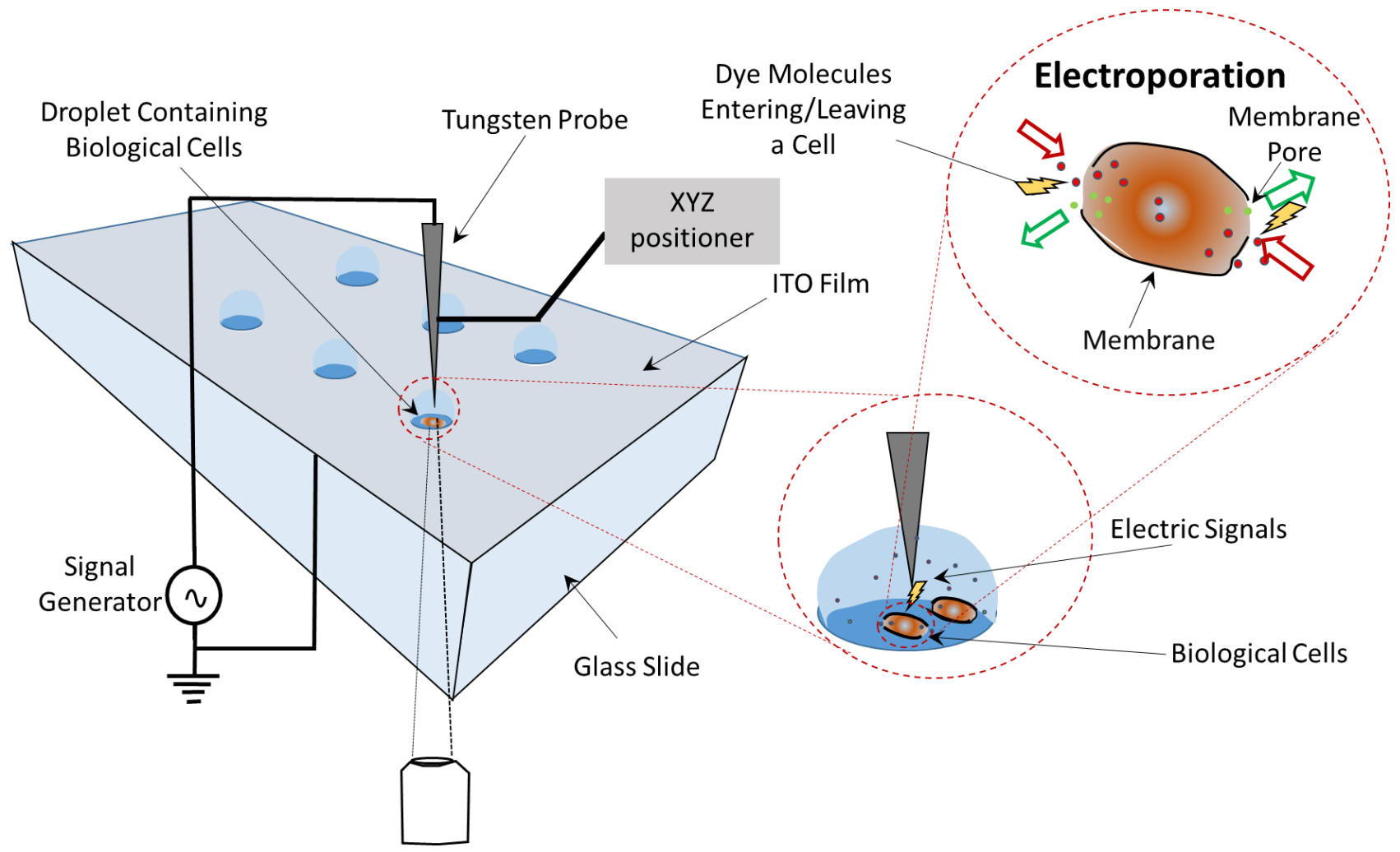

Fig 1: Schematic of the electroporation setup along with an illustration of the process through which dye molecules enter and leave cell membranes through transient pores.

\section{Materials and Methods}

The pin electrode (Microprobes) comprises of a tapered tungsten rod with a tip diameter between 3-5 microns and a shaft diameter of 250 microns. The tungsten tip is exposed directly to the liquid, but the long shaft is electrically insulated with a thin layer of parylene. The rigidity of the setup was enhanced by inserting the thin shaft into a custom designed 3D printed (Asiga MAX) probe holder. The plate electrode consists of a transparent, but highly conductive film of indium tin oxide on a glass substrate (Sigma-Aldrich). Electrical contacts were established to the electrodes using wire clamps and conductive silver epoxy.

The indium tin oxide (ITO) plate electrode is placed on an inverted microscope, and the tungsten pin electrode is mounted on a micro positioner stage which allows precise XYZ spatial control. The sinusoidal electrical signal with adjustable AC frequency was provided by a function generator (Agilent 33521A). The experimental setup containing various individual components is clearly demonstrated in Fig $2 \mathrm{a}$. 
(a)

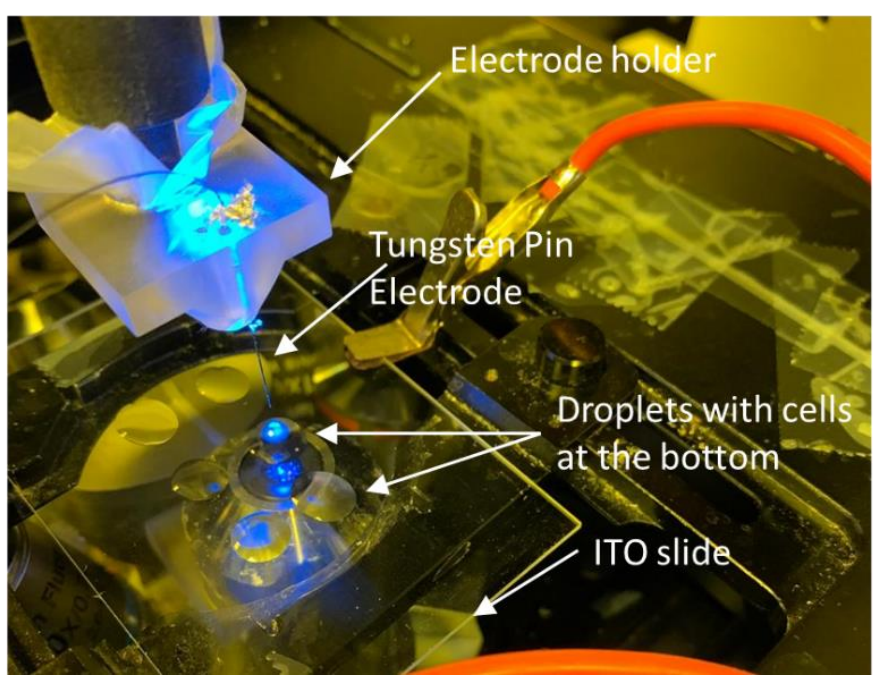

(b)

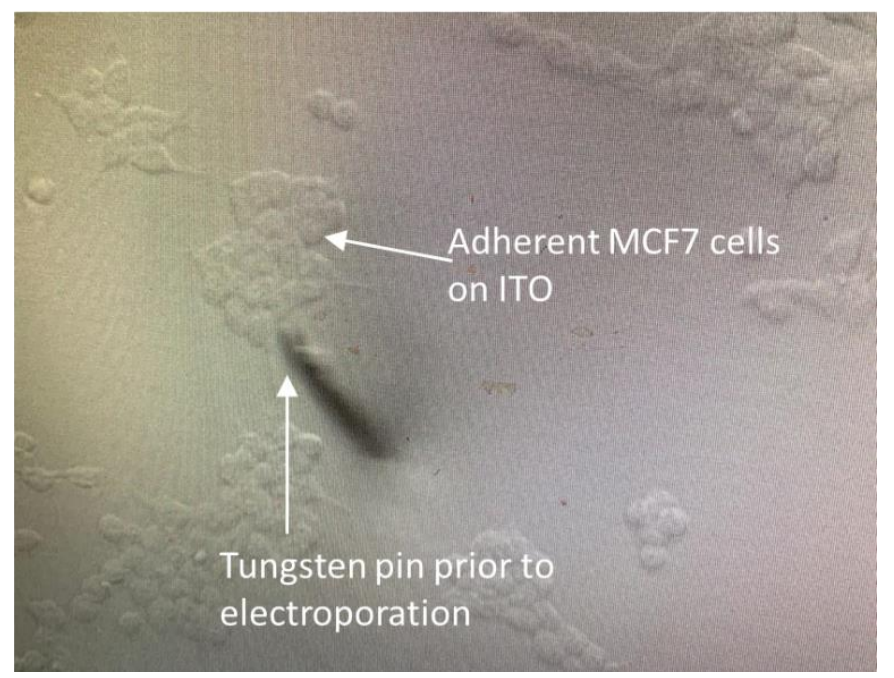

Fig 2: (a) Picture of the experimental setup consisting of tungsten pin electrodes and the planar ITO electrodes with multiple droplets on a single slide. (b) Brightfield image of a tungsten pin electrode positioned near the cells prior to electroporation.

MCF-7 cells were cultured using DMEM medium with $10 \% \mathrm{FBS}$ at $37^{\circ} \mathrm{C}$ in a humidified incubator with $5 \% \mathrm{CO}_{2}$. For these electroporation experiments, the cells must be in an adherent state, as shown in Fig $2 \mathrm{~b}$. Hence, it was necessary to plate the MCF-7 cells 24 hours prior to start of the electroporation process on the ITO coated glass slides.

Immediately before the electroporation process, the DMEM cell culture media was replaced with a low conductivity buffer solution prepared by adding $0.1 \mathrm{~g} / \mathrm{L}$ glucose and $95 \mathrm{~g} / \mathrm{L}$ sucrose to DI water. The conductivity was adjusted to 140 $\mathrm{mS} / \mathrm{m}$ with PBS, verified using a conductivity meter and the $\mathrm{pH}$ was adjusted to 7.4 with $\mathrm{NaOH}$.

To study release of the calcein AM dye from the intracellular space to the extracellular medium, cells were incubated in a solution of calcein $\mathrm{AM}(0.5 \mu \mathrm{L}$ of $4 \mathrm{mM}$ solution per $\mathrm{mL}$ of culture media) for $10 \mathrm{~min}$. Immediately after, the solution was replaced with fresh growth media. The propidium iodide (PI) solution was directly added to the electroporation buffer prior to the experiments at a concentration of $1 \mathrm{mg} / \mathrm{mL}$. After demonstrating the transfer of calcein AM and PI molecules through the cell membrane, the pin-plate electrode was used to transfect MCF-7 with a plasmid PCMV-GFP. All fluorescence images/videos were obtained with a $20 \mathrm{X}$ objective on an inverted microscope and quantified using ImageJ.

\section{Results and Discussion}

The stationary electric field between the pin electrode and the ITO coated substrate is determined using 2D finite element simulations with COMSOL Multiphysics. The geometry of the structure and the electrical parameters are set to match the parameters used experimentally. The magnitude of the field is represented by the colour plot with a maximum value of 3.17 $\mathrm{x} 10^{5} \mathrm{~V} / \mathrm{m}$ around the edges of the pin electrode, and it decays rapidly towards the ITO counter electrode, as shown in Fig 3. The profile of the electric field along the cross-section line $\mathrm{AB}$ is plotted to demonstrate single cell electroporation selectivity as a result of rapid electric field decay in the xy-plane. The line $A B$ is at a distance of 3 microns away from the bottom of the substrate to correspond to the top of the cell surface. Targeted electroporation can be achieved on two/three cell clusters without affecting the neighbouring cells because of the rapid decay in electrical field with spatial distance. 

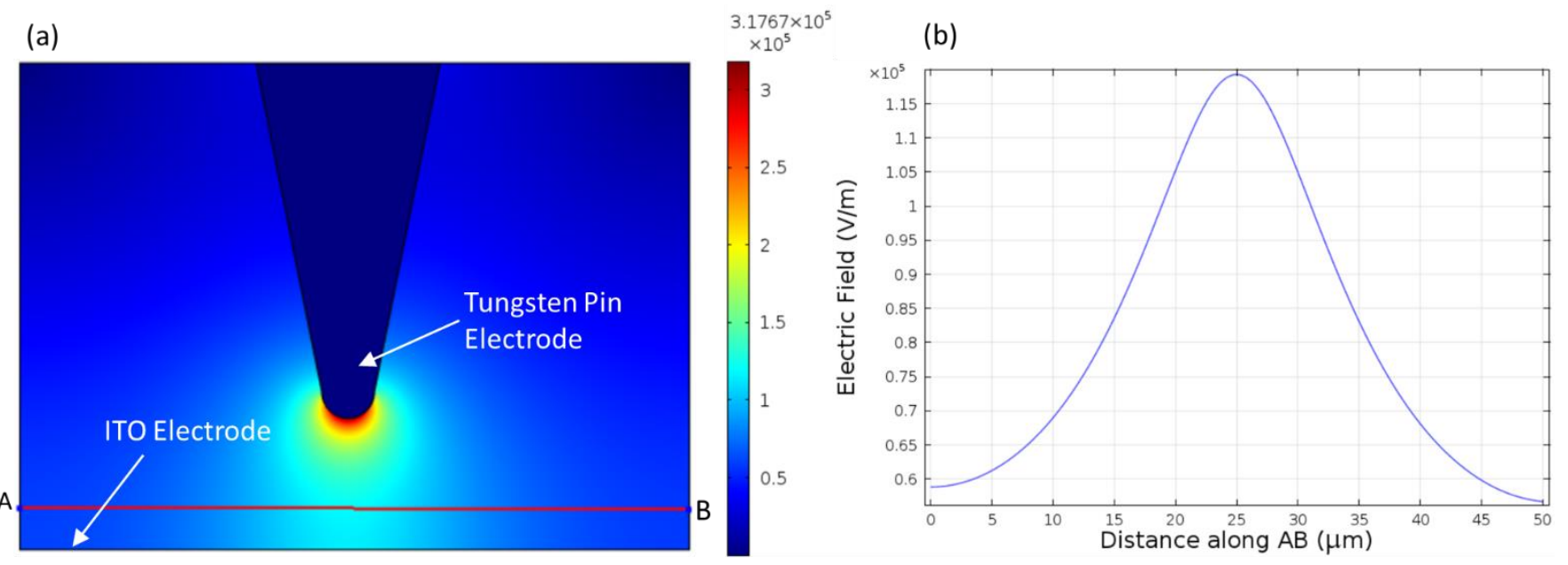

Fig 3: Finite element simulation of the Pin-Plate electrode setup for a voltage of $1.5 \mathrm{~V}_{\mathrm{pk}}$. (a) Color plot shows the electric field magnitude (V/m). (b) Line plot shows the electric field magnitude (V/m) along the cross-section line AB.

Electroporation of MCF-7 cells were initially conducted using calcein AM and propidium iodide (PI), to record the transport of molecules using fluorescence microscopy. The sinewave AC frequency was varied to study its effect on electroporation, but the electric voltage amplitude was kept constant at $1.5 \mathrm{~V}_{\mathrm{pk}}$, and the total exposure time was set to a duration of 1 second.

Upon electroporation, calcein AM diffused from the intracellular medium to the extracellular medium through the pores created on the membrane, and this was quantified using fluorescence emission at 520nm. Calcein AM was loaded into viable MCF-7 cells prior to electroporation, but immediately after electroporation, a clear decrease in fluorescence was observed in the intracellular medium due to its release into the surrounding buffer. Figure 4a also shows the dependence of calcein AM release with electric field frequency. A frequency of $10 \mathrm{kHz}$ results in a large outward flux of calcein AM, as seen by the drop in fluorescence intensity when compared to the baseline at time zero. Whereas, at a higher frequency of $1 \mathrm{MHz}$, most of the calcein AM is retained within the cell, which is an indication of smaller pore sizes.

Unlike calcein AM, PI diffuses from the extracellular medium into the cytoplasm, and PI eventually binds to DNA molecules and fluoresces at $630 \mathrm{~nm}$. A clear increase of fluorescence was observed in the intracellular space due to the entry of the dye. Figure $4 \mathrm{~b}$ shows the dependence of PI influx on electric field frequency. A frequency of $10 \mathrm{kHz}$ results in a larger inward flux of PI, as seen by the rapid increase in fluorescence intensity when compared to the baseline at time zero.

It is well understood that the electropermeabilization of biological membranes is dependent on AC field frequency [5]. Lower AC electric field frequency can cause substantial disarrangement of cell membranes, resulting in greater cell destruction. This can be explained by the amplified membrane field resulting from an electrical potential across a $5 \mathrm{~nm}$ thin cytoplasmic membrane [6]. The membrane field $\left(E_{m}\right)$ can be written as a function of the field frequency $(\omega)$ :

$$
E_{m}=\frac{1.5(R / d)|E| \cos \theta}{1+j \omega \tau}
$$

The factor $R / d$ is the ratio of the cell radius $R$ to the membrane thickness $d, \theta$ is the polar angle with respect to the field direction and $\tau$ is the time constant. 
(a)

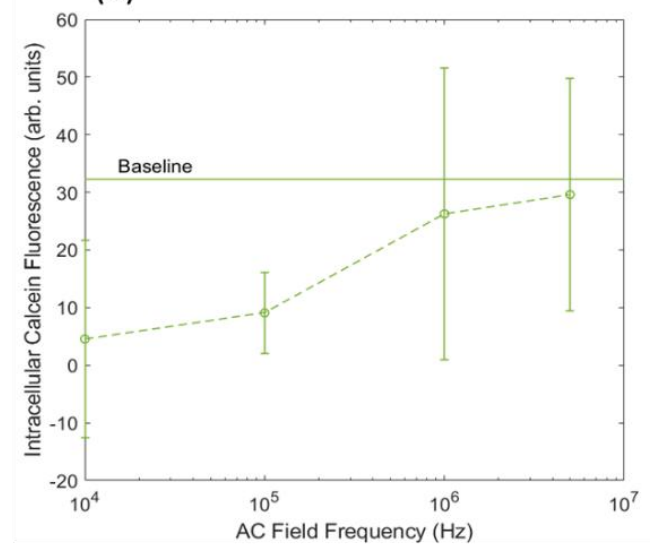

(c)

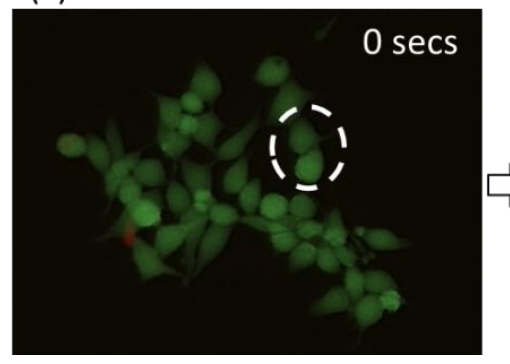

(b)

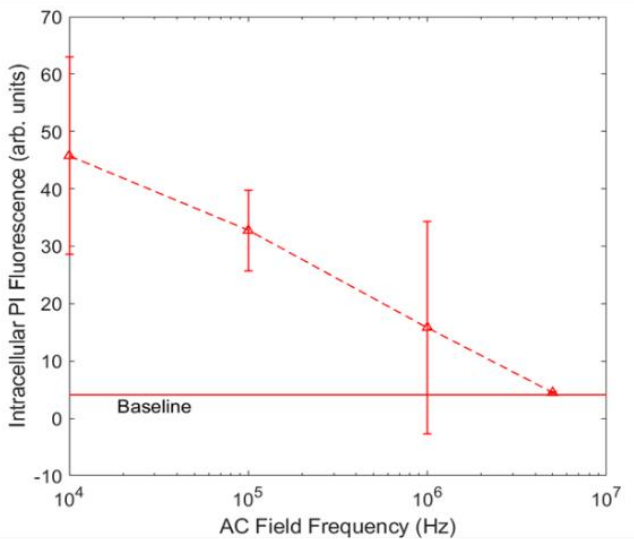

(e) (d)

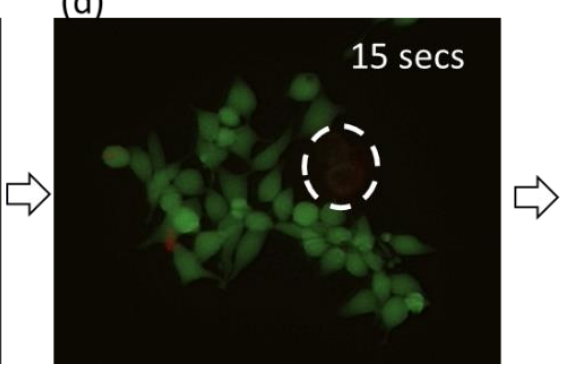

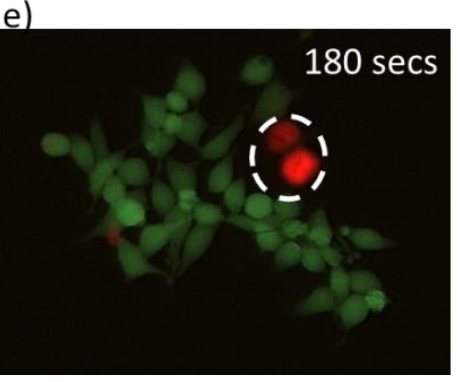

Fig 4: Fluorescence measurements on MCF-7 cells after electroporation with calcein AM (green) and PI (red). (a) AC frequency dependency showing less calcein AM release through pores with increase in frequency. As a result, at steady state, higher intracellular calcein AM is detected at steady state at higher frequency. The green baseline represents the average calcein AM fluorescence at time

zero. (b) AC frequency dependency showing less PI entry through pores at high frequency. As a result, lower intracellular PI is detected at steady state at higher frequency. Error bars indicate standard deviation for 4 cells. The red baseline represents the average PI fluorescence at time zero. (c) The tungsten probe is positioned above the encircled cells pre-stained with calcein AM. (d) Intracellular calcein AM is released 15 secs after application of a $100 \mathrm{kHz}$ sinewave. (e) Intracellular PI increases significantly after 180 secs of 100 $\mathrm{kHz}$ sinewave application.

An example of encircled cells pre-stained with calcein AM is shown in Fig 4c. This is followed by Fig 4d, which shows calcein AM release after 15 secs of $100 \mathrm{kHz}$ sinewave application with a tungsten pin-electrode (not visible). In Fig 4e, intracellular PI is shown to increase significantly after 180 secs. A longer time of 180 secs seen for PI incorporation compared to 15 secs for calcein AM release can be attributed to the period for intercalation of PI into the DNA base pairs. Despite positioning the pin electrode over a dense cluster of cells, this electrode setup has demonstrated targeted electroporation of just two cells without affecting the neighbouring cells. This can be attributed to the rapid decay in the electrical field with spatial distance, as validated by the finite element simulations.

After demonstrating the transfer of calcein AM and PI molecules through the cell membrane, the pin-plate electrode was used to transfect MCF-7 with PCMV-GFP at a frequency of $1 \mathrm{MHz}$ for a total duration of 1 second, as shown in Fig 5a. It is clear from Equation 1 that the magnitude of the external field in the membrane drops with increasing frequency, thereby significantly minimizing cell membrane damage. With the aim to maximize cell viability and minimize cellular damage, a frequency of $1 \mathrm{MHz}$ was employed for electroporation-based cell transfection. GFP expression shown in Fig 5b, was observed approximately 48 hours post-electroporation, which demonstrates successful transfection while maintaining cell viability and proliferation capacity. 
(a)

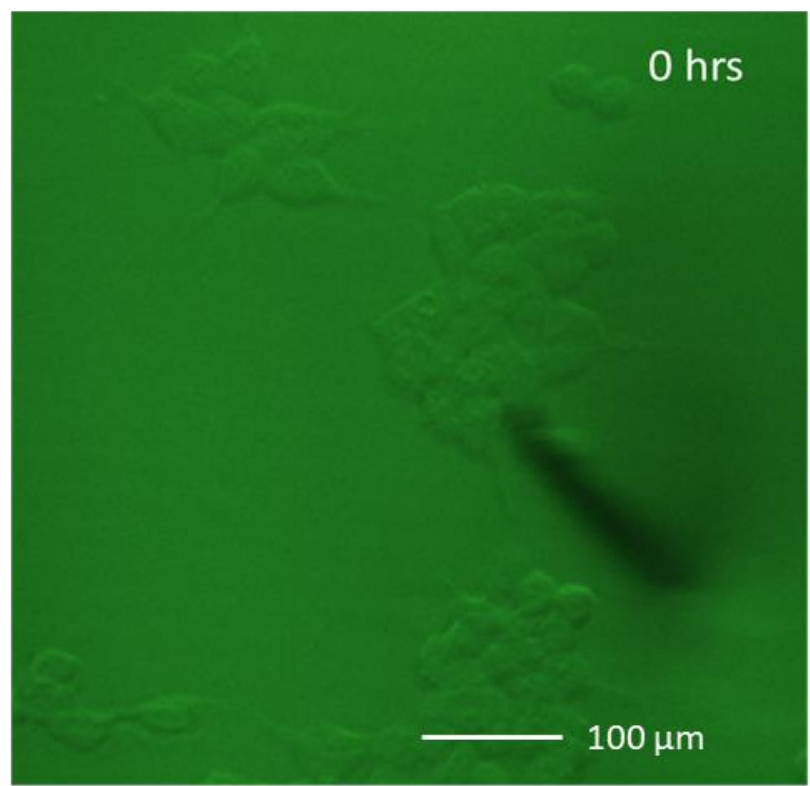

(b)

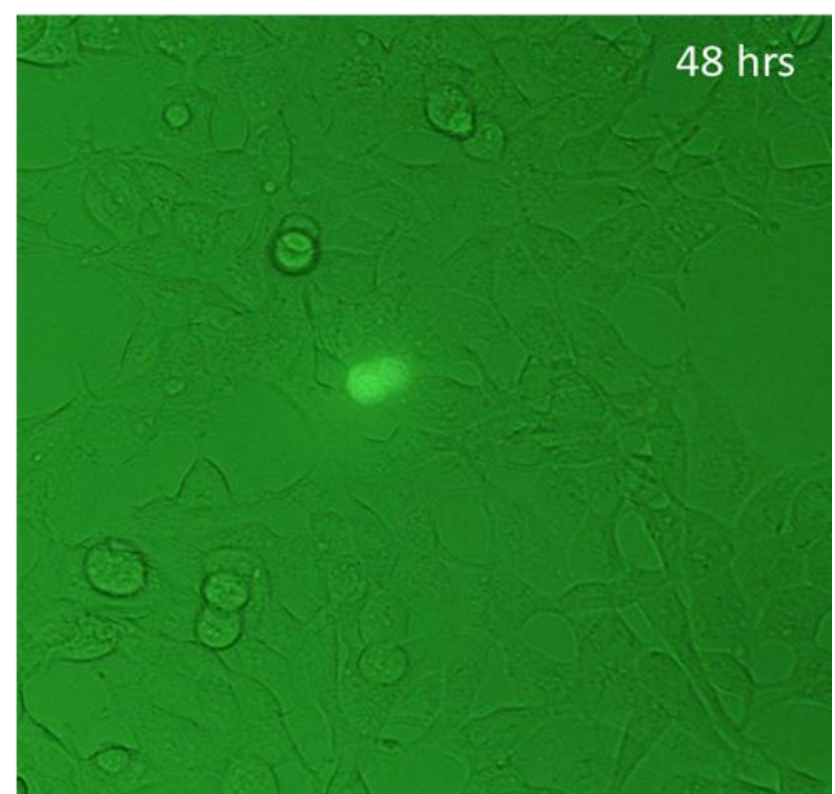

Figure 5: Green fluorescent protein (GFP) expression with a CMV promoter. (a) Fluorescence measurements before electroporation. (b) GFP fluorescence 48 hours post transfection and after incorporation of plasmid DNA.

\section{Conclusion}

This simple approach using an electrode pin inserted into microdroplets enable rapid sequential electroporation. The low volume of liquid within these droplets also limits expensive reagent cost. Continuous monitoring of the electroporation process was possible through the transparent ITO electrode by means of microscopy. In addition, the underlying ITO coated glass substrate allows cells to be cultured directly on them post-electroporation. The demonstrated pin-plate electrode system on a single chip can be used as a platform for targeted delivery of regents to two/three cell clusters and even single cells.

\section{References}

[1] S. Kim and J. Eberwine, "Mammalian cell transfection: The present and the future". Anal. Bioanal. Chem., vol. 397, no. 8, pp. 3173-3178, 2010.

[2] H. Potter and R. Heller, "Transfection by Electroporation". Curr Protoc Mol Biol., vol. 121, no. 1, pp. 9-3, 2018.

[3] T. Kotnik, L M Mir, K Flisar, M Puc and D Miklavcic "Cell membrane electropermeabilization by symmetrical bipolar rectangular pulses: Part I. Increased efficiency of permeabilization." Bioelectrochemistry, vol. 54., no. 1. pp. 83-90, 2001

[4] T. Geng, and C. Lu, "Microfluidic Electroporation for Cellular Analysis and Delivery". Lab on a Chip, vol 13, no. 19, pp. 3803-3821, 2013.

[5] T. García-Sánchez, C. Merla , J. Fontaine, A. Muscat and L. M. Mir "Sine wave electropermeabilization reveals the frequency-dependent response of the biological membranes." Biochimica et Biophysica Acta (BBA)-Biomembranes, vol. 1860 , no. 5 , pp. 1022-1034, 2018.

[6] A. Menachery, and R. Pethig, "Controlling cell destruction using dielectrophoretic forces." in IEE ProceedingsNanobiotechnology, vol. 152, no. 4, pp. 145-149, 2005. 